\title{
Extension of Wavelength Range in Absolute Intensity Calibration of Space-Resolved EUV Spectrometer for LHD Diagnostics*)
}

\author{
Chunfeng DONG ${ }^{1)}$, Shigeru MORITA ${ }^{1,2)}$, Motoshi GOTO ${ }^{1,2)}$ and Erhui WANG ${ }^{2)}$ \\ 1) National Institute for Fusion Science, Toki, Gifu 509-5292, Japan \\ ${ }^{2)}$ Department of Fusion Science, Graduate University for Advanced Studies, Toki, Gifu 509-5292, Japan
}

(Received 9 December 2011 / Accepted 25 June 2012)

\begin{abstract}
A space-resolved extreme ultraviolet (EUV) spectrometer has been upgraded by extending the wavelength range to 30-650 $\AA$ to explore impurity line emissions existing at shorter and longer wavelength sides. The absolute intensity calibration is implemented for measurement in the extended wavelength based on bremsstrahlung profiles simultaneously measured in EUV and visible ranges. For the purpose a wider entrance slit of $200 \mu \mathrm{m}$ and a wider space-resolved slit of $1.0 \mathrm{~mm}$ are adopted to increase the number of photons to the spectrometer. As a result, the bremsstrahlung intensity can be enhanced by order of magnitude. A centrally peaked high-density discharge at $n_{\mathrm{e}}(0) \geq 10^{14} \mathrm{~cm}^{-3}$ is also used for the accurate calibration. Thus, the calibration becomes possible, even in longer wavelength side at $\lambda \geq 400 \AA$. The result at shorter wavelength range of 30-90 shows a flat calibration factor, suggesting sudden changes of holographic grating efficiency and CCD detection efficiency, while the result at longer wavelength side of $\lambda \geq 400 \AA$ shows a simple extension of the previous calibration factor.
\end{abstract}

(C) 2012 The Japan Society of Plasma Science and Nuclear Fusion Research

Keywords: EUV bremsstrahlung, grating efficiency, absolute intensity calibration

DOI: $10.1585 /$ pfr.7.2402139

\section{Inroduction}

Extreme ultraviolet (EUV) spectroscopy plays an essential role in the impurity diagnostics of high-temperature plasmas, since impurities are ionized into highly charge states and wavelengths emitted from such impurity ions become shorter. A space-resolved EUV spectrometer working in wavelength range of $60-400 \AA$ has been installed in Large Helical Device (LHD) to measure radial profiles of impurity line emissions and bremsstrahlung continuum for the impurity transport study and effective ion charge $\left(Z_{\mathrm{eff}}\right)$ measurement, respectively [1]. For these purposes the absolute intensity calibration of the space-resolved EUV spectrometer is required. Due to a qualitative limitation of conventional methods for the absolute intensity calibration using synchrotron orbital radiation source and branching ratio technique, a new method based on the bremsstrahlung continuum measurement in EUV and visible range has been developed, and could be successfully applied to calibration of the space-resolved EUV spectrometer in wavelength range of 80-400 $\AA$ [2].

Recently, the wavelength range of the space-resolved EUV spectrometer was extended to $30-650 \AA$ to measure the first resonance lines of BIV (61.1 $\AA$ ), BV (48.6 $)$, CV (40.3 $\AA$ ) and CVI (33.7 $\AA$ ) as well as NeVII (465.2 $\left.2 \mathrm{~s} 2 \mathrm{p}-2 \mathrm{~s}^{2}\right)$ and OV (629.7 $\AA$ : $\left.2 \mathrm{~s} 2 \mathrm{p}-2 \mathrm{~s}^{2}\right)$. As dominant impurity in LHD is only carbon, measurement of the first resonance lines of $\mathrm{CV}$ and CVI are essentially impor-

author's e-mail: dong.chunfeng@nifs.ac.jp

*) This article is based on the presentation at the 21st International Toki Conference (ITC21). tant for studying the edge impurity transport. The impurity line emissions of BIV and BV appeared after the boronization are also useful for studying the boron behavior. The edge electron temperature measurement using impurity line intensity ratio, e.g., NeVII (97.5 ̊: 2s3p$2 \mathrm{~s}^{2}$ )/NeVII (465.2 $\AA$ : $2 \mathrm{~s} 2 \mathrm{p}-2 \mathrm{~s}^{2}$ ), is also possible. In order to quantitatively analyze the spectral data obtained from the EUV spectrometer, the absolute intensity calibration is carried out in the extended wavelength range using the same method as reported in Ref. [2].

\section{Experimental Arrangements}

LHD discharges initiated by electron cyclotron heating $(\mathrm{ECH})$ are sustained by three tangential neutral beams with beam energy of $180 \mathrm{keV}$. High density plasmas in range of $n_{\mathrm{e}}(0) \geq 10^{14} \mathrm{~cm}^{-3}$ are produced by multihydrogen ice pellet injection using repetitive pellet injector [3] in magnetic axis position of $R_{\mathrm{ax}}=3.60 \mathrm{~m}$ at high magnetic field operation of $2.75 \mathrm{~T}$. Relatively small-sized and low-speed pellets are repetitively injected to avoid too much perturbation to the core plasma. Typical size and velocity of the cylindrical pellets are $2.5 \mathrm{~mm} \times 2.5 \mathrm{~mm}$ (diameter $\times$ length) and $250 \mathrm{~m} / \mathrm{s}$, respectively.

Vertical profiles of the bremsstrahlung continuum are simultaneously observed in the EUV and visible wavelength ranges using the flat-field space-resolved EUV spectrometer and an astigmatism-corrected Czerny-TurnerType visible spectrometer, respectively. The EUV spectrometer consists of an entrance slit, a space-resolved slit, 


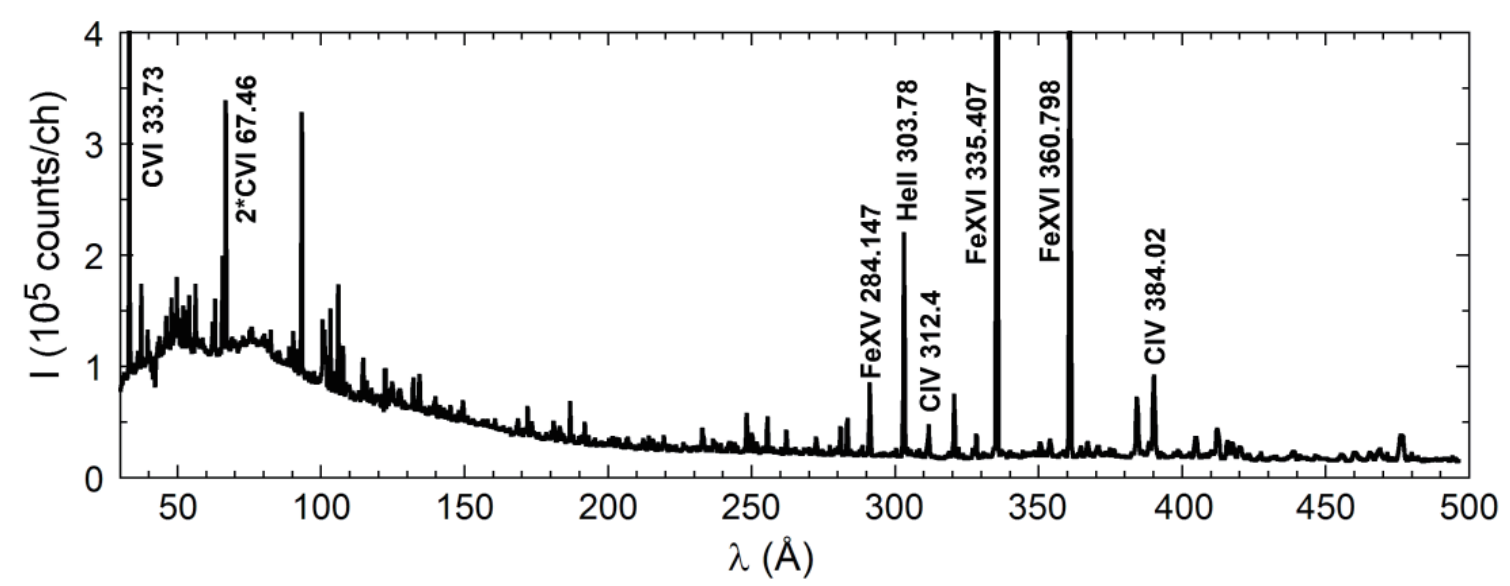

Fig. 1 Typical EUV spectrum observed from multi-hydrogen pellet injected plasma. Data are composed of several discharges with different plasma parameters.

a gold-coated concave varied-line-spacing (VLS) laminartype holographic grating (1200 grooves $/ \mathrm{mm}$ ) and a chargecoupled device $(\mathrm{CCD})$ detector. In order to increase the incident photons, a wider entrance slit of $200 \mu \mathrm{m}$ in width and a wider space-resolved slit of $1.0 \mathrm{~mm}$ in width are adopted in the present study. The EUV spectrometer is installed in LHD perpendicular to the toroidal magnetic field at horizontally elongated plasma cross section. The vertical observation range of the EUV spectrometer is nearly $0.55 \mathrm{~m}$, and then, half of the LHD plasma can be observed at the horizontally elongated plasma cross section. The EUV bremsstrahlung profile from the upper half of LHD plasmas is usually measured for the absolute intensity calibration.

Meanwhile, the visible spectrometer with 44 parallel fiber arrays has an observation chord tilted by $6^{\circ}$ from the direction perpendicular to the toroidal magnetic field and the fiber arrays cover the full vertical range of LHD plasmas from $-0.6 \mathrm{~m}$ to $0.6 \mathrm{~m} \mathrm{[4]}$. A 300 grooves $/ \mathrm{mm}$ grating enables the visible spectrometer to measure the spectra in wavelength range of 4575-6000. . The absolute intensity calibration of the visible spectrometer system has been accurately done using an integrating sphere. The profiles of electron density and temperature are measured with a Nd:YAG laser Thomson scattering system, in which the laser path is arranged along the equatorial plane at a horizontally elongated plasma cross section [5]. The Thomson scattering system has been optimized to measure the temperature range of $50 \mathrm{eV}$ to $10 \mathrm{keV}$ [6].

\section{Optimization of EUV Bremsstrahlung Measurement}

Since the intensity of EUV bremsstrahlung increases with electron density as seen in the previous result [2], the number of EUV photons entering on the CCD detector is sufficient for the calibration in high-density discharges, in particular, at short wavelength range below $200 \AA$. Wave- length of the space-resolved EUV spectrometer is changed by moving the position of CCD detector through external control system connected to LAN network. The modification of the spectrometer system is done for the wavelength scanning system. The spectrometer is upgraded by adding the second movement stage to the CCD detector to make wider movement distance, which leads to the extension of wavelength range to $30-650 \AA$. Typical EUV spectrum in wavelength range of $30-500 \AA$ observed from high-density plasma with multi-hydrogen ice pellet injection is shown in Fig. 1. A special attention should be paid to the short wavelength range below $80 \AA$. The measured bremsstrahlung intensity stops increasing at $\lambda=80 \AA$ and takes roughly constant at $\lambda<80 \AA$, whereas the bremsstrahlung intensity from plasma continuously increases even at $\lambda<80 \AA$ [7]. It strongly suggests that the laminar-type holographic grating efficiency or CCD detector efficiency suddenly change at $\lambda<80 \AA$.

On the other hand, the bremsstrahlung intensity becomes weak in long wavelength side, in particular, at $\lambda>$ $400 \AA$ A In order to measure enough EUV bremsstrahlung intensity in the long wavelength side, a entrance slit of $100 \mu \mathrm{m}$ width and a space-resolved slit of $0.5 \mathrm{~mm}$ width are replaced by $200 \mu \mathrm{m}$ and $1.0 \mathrm{~mm}$, respectively, to increase the number of photons entering into the spectrometer. The vertical profile of EUV bremsstrahlung continuum measured at $379 \AA$ in high-density discharges is compared between the two different slit cases, as plotted in Fig. 2 . Here, it should be noticed that the two profiles are measured from discharges with different densities and positions of the magnetic axis, i.e., $n_{\mathrm{e}}=22 \times 10^{13} \mathrm{~cm}^{-3}$ and $R_{\mathrm{ax}}$ $=3.75 \mathrm{~m}$ for original slit case and $n_{\mathrm{e}}=11 \times 10^{13} \mathrm{~cm}^{-3}$ and $R_{\mathrm{ax}}=3.60 \mathrm{~m}$ for wide slit case. Taking the density effect into account, which can enlarge the intensity by a factor of 4 at $n_{\mathrm{e}}=22 \times 10^{13} \mathrm{~cm}^{-3}$, therefore, actual bremsstrahlung intensity entering into the spectrometer can be increased by order of magnitude, taking into account the density difference between the two discharges. 


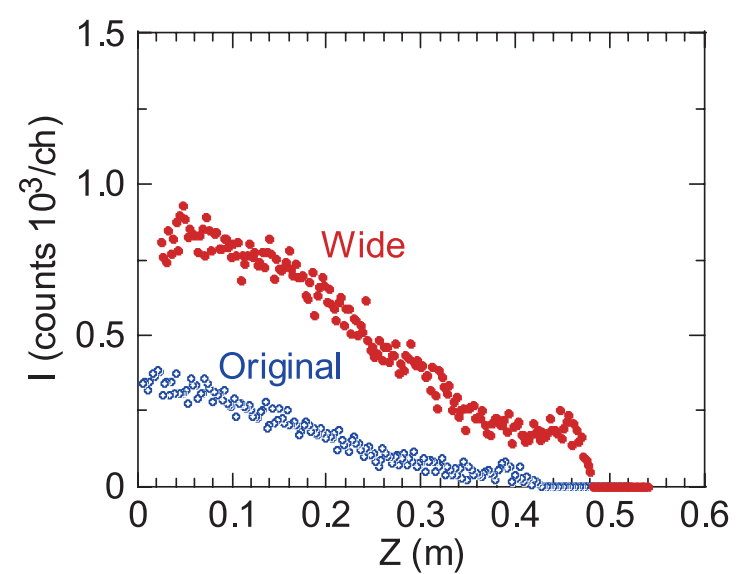

Fig. 2 Vertical profiles of EUV bremsstrahlung continuum at $379 \AA$ measured with original (entrance: $100 \mu \mathrm{m}$ and space-resolution: $0.5 \mathrm{~mm})$ and wide $(200 \mu \mathrm{m}$ and $1.0 \mathrm{~mm}$ ) slits.

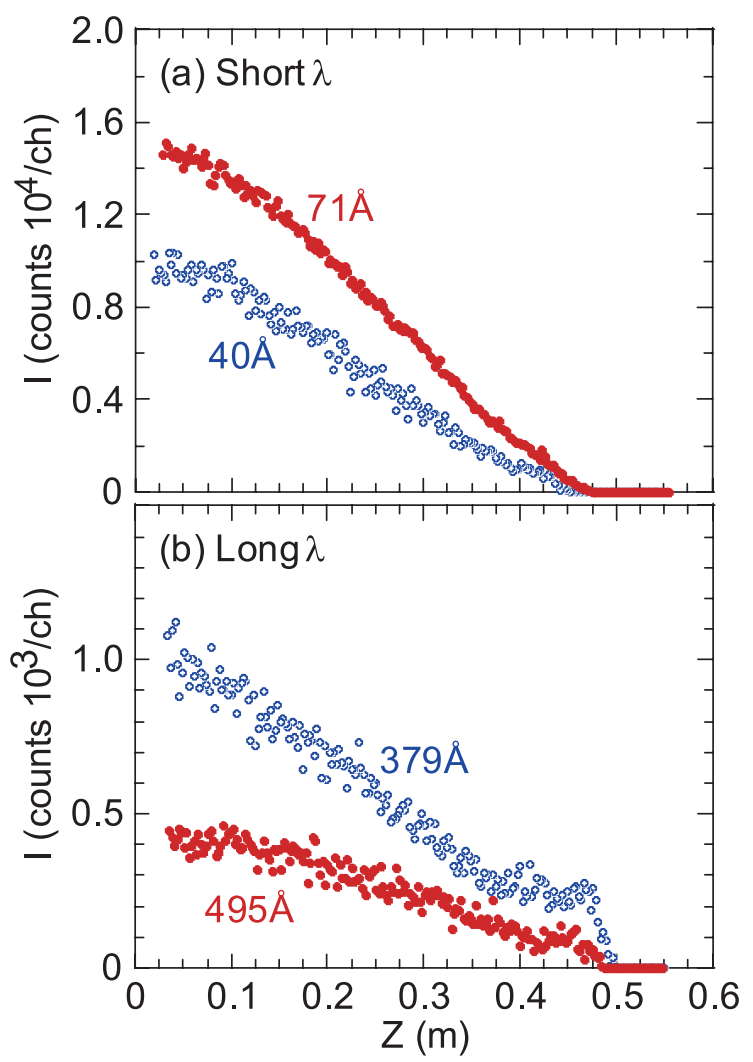

Fig. 3 Vertical profiles of EUV bremsstrahlung continuum at (a) short wavelength side and (b) long wavelength side. Data include effect of different plasma parameters based on considerably large shot-to-shot variation.

The vertical profiles of EUV bremsstrahlung continuum are plotted in Figs. 3 (a) and (b) for short and long wavelength ranges, respectively. As seen in Fig. 3 (a), the bremsstrahlung intensity measured at $40 \AA$ is weaker than that measured at $71 \AA$, showing the same result as Fig. 1 . Since two profiles in Fig. 3(b) are observed from discharges with different parameters, the difference in inten- sity between $379 \AA$ and $495 \AA$ is enlarged by a factor of 1.6 , arising from the different density and temperature. Whatever the case, a centrally peaked EUV bremsstrahlung profile is observed for all the wavelength ranges. The peaked profile desired for good Abel inversion further enhances the accuracy of absolute intensity calibration, especially in the long wavelength range.

\section{Absolute Intensity Calibration}

The continuum radiation is composed of free-free radiation and free-bound radiation known as bremsstrahlung and radiative recombination, respectively. As the electron temperature is fairly high in fusion plasmas, the contribution of radiative recombination to the continuum radiation is negligible in the present wavelength ranges. In the expression of bremsstrahlung continuum radiation the power ratio of EUV to visible bremsstrahlung can be written by

$$
\begin{aligned}
\frac{p_{\text {brem_EUV }}}{p_{\text {brem_Vis }}}= & \left(\frac{\lambda_{\text {Vis }}}{\lambda_{\text {EUV }}}\right)^{2} \frac{g_{\text {ff_EUV }}}{g_{\text {ff_Vis }}} \\
& \times \exp \left[-\frac{12400}{T_{\mathrm{e}}}\left(\frac{1}{\lambda_{\mathrm{EUV}}}-\frac{1}{\lambda_{\text {Vis }}}\right)\right],
\end{aligned}
$$

where $P_{\text {brem }}$ is the bremsstrahlung radiation power in unit of $\mathrm{Wcm}^{-3} \AA^{-1}, \lambda$ is the corresponding wavelength in unit of $\AA, g_{\mathrm{ff}}$ is the free-free gaunt factor and $T_{\mathrm{e}}$ is the electron temperature in unit of $\mathrm{eV}$. Since the free-free gaunt factor has a weak dependence on electron temperature and wavelength, a computer program is utilized to calculate the gaunt factor [8]. Detailed process on the absolute intensity calibration is introduced in Ref. [2]. Then, the calibration process done in the present study is briefly.

The visible bremsstrahlung continuum at $5330 \AA$ is adopted for the absolute calibration. The profile in local emissivity of visible bremsstrahlung, $\varepsilon_{\text {brem }}$, is shown in Fig. 4 (a), which is derived from chord-integrated profile measured by the visible spectrometer through Abel inversion calculation. Magnetic surface distortion caused by the plasma pressure is considered in the calculation. The electron temperature profile is converted into the magnetic surface coordinates from the geometric coordinates using a free-boundary equilibrium code, VMEC. Based on the Eq. (1), the local emissivity profile of EUV bremsstrahlung at $54 \AA$ can be calculated from the visible bremsstrahlung local emissivity and temperature profiles. The result is shown in Fig. 4(b). The chord-integrated EUV bremsstrahlung intensity profile is then obtained by integrating the profile plotted in Fig. 4 (b) along the observation chord, as shown in Fig. 5(a). The vertical profile of EUV bremsstrahlung at $54 \AA$ measured by the spaceresolved EUV spectrometer is represented in Fig. 5(b). Taking account of the sampling time of CCD detector and the wavelength interval of the EUV spectrometer, the absolute intensity calibration factor is thus obtained by comparing the calculated chord-integrated profile with the measured one. The result is shown in Fig. 5 (c). It shows a 


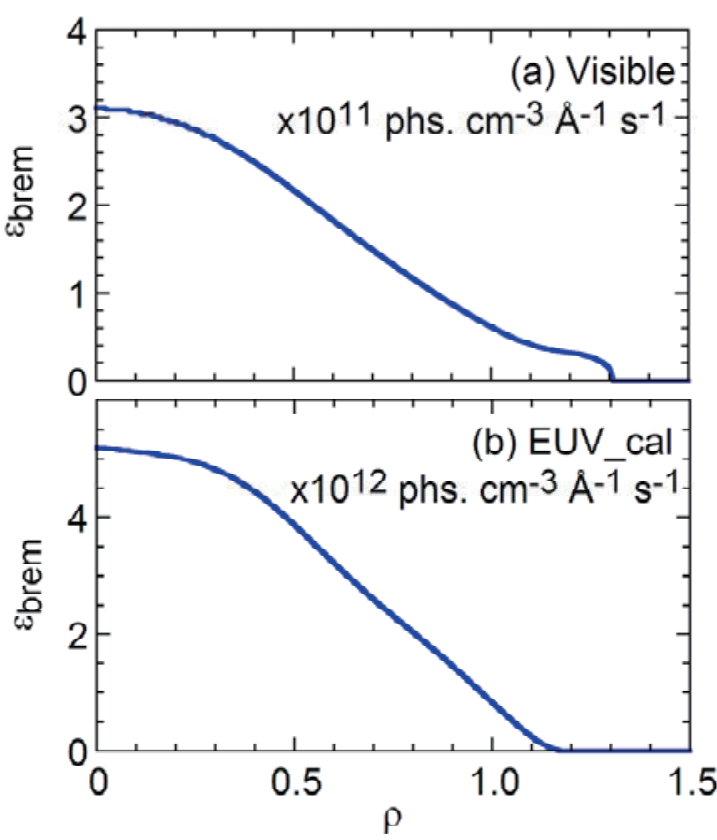

Fig. 4 (a) Local emissivity profile of visible bremsstrahlung at $5330 \AA$ derived from measured chord-integrated profile and (b) local emissivity profile of EUV bremsstrahlung at $54 \AA$ A calculated from Fig. 4 (a).

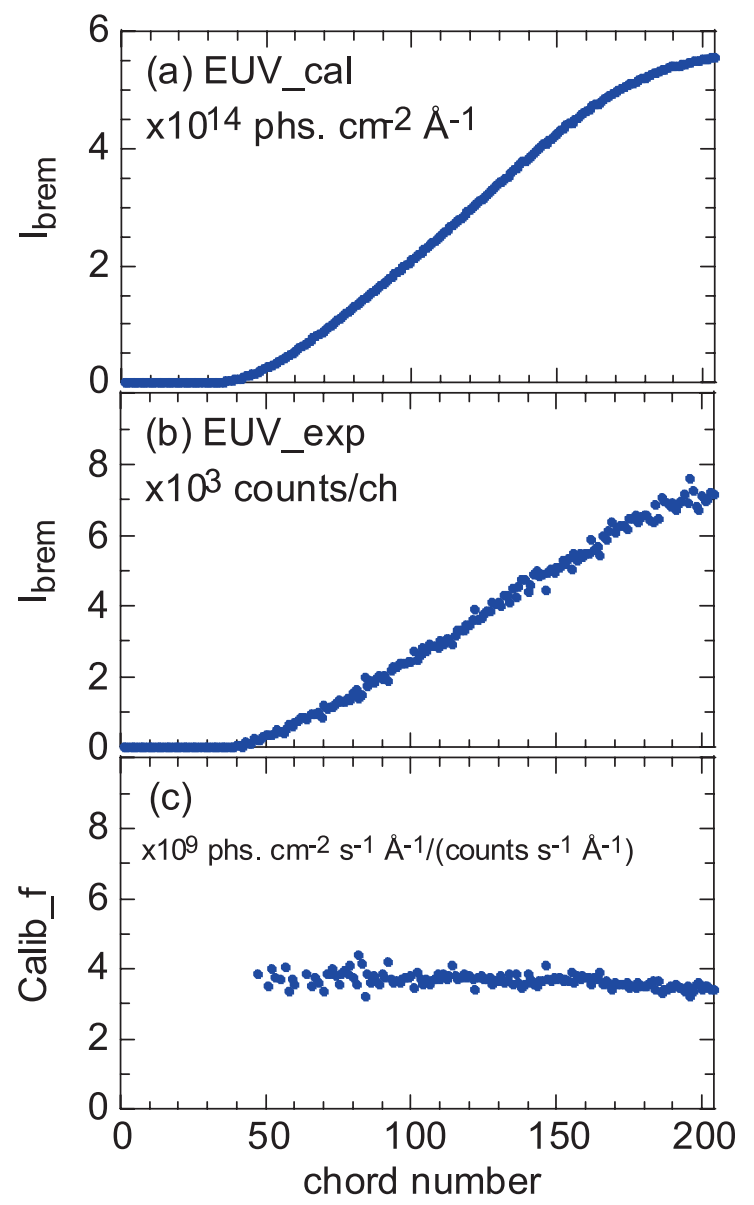

Fig. 5 (a) Calculated and (b) measured chord-integrated vertical profiles of EUV bremsstrahlung at $54 \AA$ and (c) absolute intensity calibration factor of space-resolved EUV spectrometer at $54 \AA$.

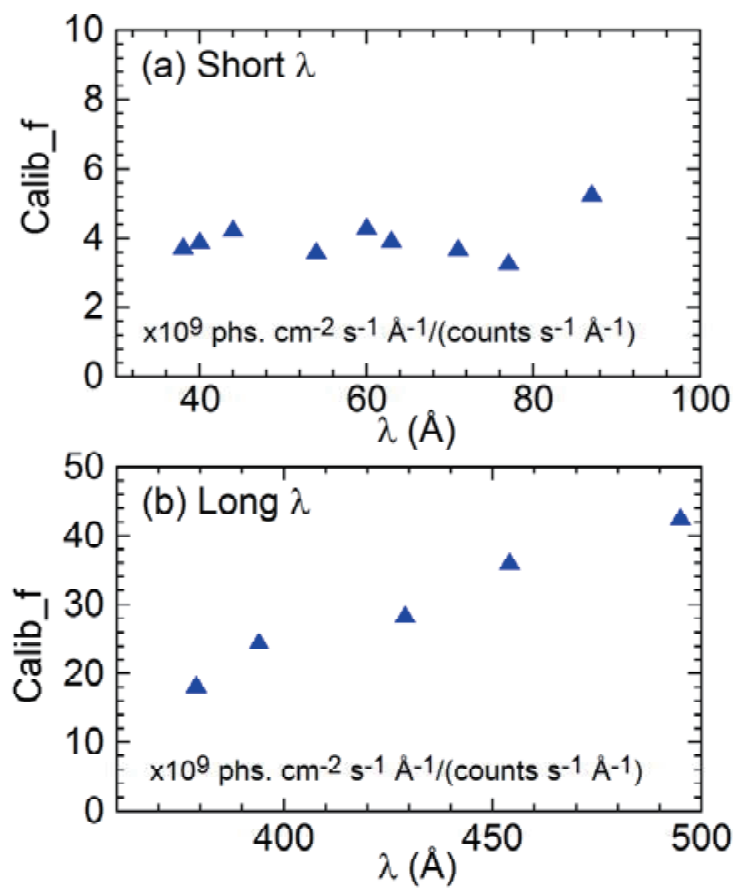

Fig. 6 Absolute intensity calibration factor of upgraded spaceresolved EUV spectrometer at (a) short wavelength side and (b) long wavelength side.

constant calibration factor along the chord number, which is consistent with the previous result. The flat calibration factor profile indicates that the reflectivity of the EUV emissions on the holographic grating is constant along the direction perpendicular to the wavelength dispersion.

The absolute intensity calibration process is applied to the extended wavelength range of the present spaceresolved EUV spectrometer at a series of discrete wavelength positions. The results are shown in Figs. 6 (a) and (b) for the short and long wavelength ranges, respectively. The present result at long wavelength side in Fig. 6(b) shows a consistent tendency with the previous result, i.e., monotonous increase with wavelength showing a simple extension of the previous calibration factor. The value of the calibration factor is reduced by order of magnitude in comparison with the previous result, since the two slits are replaced by wider ones. In contrast, the calibration factor at short wavelength in Fig. 6 (a) seems to be almost constant, in particular, at $\lambda<80 \AA$. The value of the calibration factor is only reduced to $1 / 2$ or $1 / 3$ in comparison with the previous result. The reason is not clear now, but at least the result strongly suggests sudden changes of holographic grating efficiency and CCD detection efficiency in the short wavelength range.

Here, the error of the calibration factor is estimated. One of the uncertainties is originated in the Abel inversion process. The uncertainty is estimated to be less than $5 \%$ [9]. The electron temperature measured by Thomson scattering system also brings the uncertainty, which is estimated to be less than $3 \%$. The final factor is based on the 
noise count in the CCD appeared only during discharge phase, whereas the CCD is cooled down enough. This uncertainty can be estimated to be less than $10 \%$, which becomes bigger in longer wavelength side. The detailed description of the error of the calibration factor is given in Ref. [2].

\section{Summary}

The space-resolved EUV spectrometer extended to wider wavelength range of $30-650 \AA$ is calibrated on the absolute intensity. For the purpose a wider entrance slit of $200 \mu \mathrm{m}$ and a wider space-resolved slit of $1.0 \mathrm{~mm}$ are used to increase the number of photons entering into the spectrometer, which is especially important for the calibration at long wavelength range. A centrally peaked EUV bremsstrahlung profile is also used by measuring highdensity plasmas produced by multi-hydrogen pellet injection. The result of calibration in the long wavelength side is consistent with the previous result. However, the result in the short wavelength indicates a considerable difference in comparison with the previous result. In order to make it clear, the present result is compared with theoretical calculation on the grating efficiency in near future. The CCD detection efficiency has to be also experimentally examined as a function of wavelength in EUV range.

\section{Acknowledgements}

The authors are grateful to Prof. R. Sakamoto and Dr. G. Motojima for theircooperation on the hydrogen pellet injection. The authors would like to also thank all members of the LHD experimental group for their technical supports. This work was partially carried out under the LHD project financial support (NIFS11ULPP010). This work was also partially supported by the JSPS-NRF-NSFC A3 Foresight Program in the field of Plasma Physics (NSFC: No.11261140328).

[1] C.F. Dong, S. Morita, M. Goto and H.Y. Zhou, Rev. Sci. Instrum. 81, 033107 (2010).

[2] C.F. Dong, S. Morita, M. Goto and E.H. Wang, Rev. Sci. Instrum. 82, 113102 (2011).

[3] R. Sakamoto, H. Yamada, Y. Takeiri, K. Narihara, T. Tokuzawa, H. Suzuki, S. Masuzaki, S. Sakakibara, S. Morita, M. Goto, B.J. Peterson, K. Matsuoka, N. Ohyabu, A. Komori, O. Motojima and the LHD experimental group, Nucl. Fusion 46, 884 (2006).

[4] H.Y. Zhou, S. Morita, M. Goto and M.B. Chowdhuri, Rev. Sci. Instrum. 79, 10F536 (2008).

[5] K. Narihara, I. Yamada, H. Hayashi and K. Yamauchi, Rev. Sci. Instrum. 72, 1122 (2001).

[6] I. Yamada, K. Narihara, H, Funaba, H. Hayashi, T. Kohmoto, H. Takahashi, T. Shimozuma, S. Kubo, Y. Yoshimura, H. Igami and N. Tamura, Rev. Sci. Instrum. 81, 10D522 (2010).

[7] C.F. Dong, S. Morita, M.B. Chowdhuri and M. Goto, Plasma Fusion Res. 6, 2402078 (2011).

[8] P.J. Storey and D.G. hummer, Comput. Phys. Commun. 66, 129 (1991).

[9] H.Y. Zhou, S. Morita, M. Goto and C.F. Dong, J. Appl. Phys. 107, 053306 (2010). 